\title{
Developmental establishment of epigenotype: a role for dietary fatty acids?
}

\author{
Robert A. Waterland and Marie-Therese Rached \\ Departments of Pediatrics and Molecular and Human Genetics, Baylor College of Medicine, USDA Children's Nutrition \\ Research Center, Houston, Texas, USA
}

Abstract

Human epidemiologic data indicate that nutrition during prenatal and early postnatal development can affect susceptibility to various chronic diseases in adulthood (the developmental origins hypothesis). Controlled studies in animal models corroborate that nutritional exposures during critical periods of development wield lasting influences on gene expression and metabolism, but our understanding of the fundamental biological mechanisms underlying such phenomena remains rudimentary. Nutritional influences on the developmental establishment of epigenetic gene regulatory mechanisms could link early nutrition to adult chronic disease susceptibility. Just as genetic variation contributes to individual susceptibility to chronic disease, it is increasingly evident that so too does epigenetic variation. Very little is known about the factors that contribute to interindividual variation in epigenotype. It is critically important to determine whether nutrition and other environmental influences during development affect the establishment of human epigenotype. Most previous studies of nutritional influences on epigenetic regulation have focused on nutrients known to affect one-carbon metabolism. For example, supplementation during development with methyl donors including folic acid and vitamin $\mathrm{B}_{12}$ appears to induce DNA hypermethylation at specific loci by increasing the flux through the DNA methylation pathway. This article will consider the mechanisms by which fatty acid nutrition during prenatal and early postnatal nutrition might affect the establishment of epigenetic gene regulatory mechanisms. Choline, a component of the phospholipid phosphatidylcholine, stands at the intersection of lipid and one-carbon metabolism; upon conversion to betaine, its three methyl groups are available to one-carbon metabolism. Potentially much more important than providing methyl groups for DNA methylation, dietary fatty acids could affect the establishment of epigenetic mechanisms by stimulating transcription of specific genes during critical developmental windows. For example, the peroxisome proliferator activated receptor- $\alpha$ (PPAR- $\alpha)$ transcription factor is activated by a variety of fatty acids to transactivate specific genes. PPAR- $\alpha$ mediated transcriptional activation during critical ontogenic periods could impede epigenetic silencing of genes involved in fatty acid metabolism. Lastly, recent data indicate that lipids and lipoprotein components interact directly with chromatin structure to influence gene expression. Hence, intake of specific dietary fatty acids during development could induce persistent changes in gene expression by altering the establishment of epigenetic mechanisms.

Keywords: DNA methylation, Developmental origins, Epigenetics, Metabolic imprinting, Nutrition

Abbreviations: Aox: acyl-coenzyme A oxidase; ApoE: apolipoprotein E; E: embryonic day; FFA: free fatty acid; GR: glucocorticoid receptor; HDL: high-density lipoprotein; HSP: heat-shock protein; LDL: low-density lipoprotein; P: postnatal day; PPAR: peroxisome proliferator activated receptor; PPRE: peroxisome proliferator response element; PUFA: polyunsaturated fatty acid; $R X R$ : retinoid $X$ receptor; VLDL: very low-density lipoprotein.

\section{Fatty acids and metabolic imprinting}

7 he developmental origins hypothesis proposes that the pathogenesis of many diet-related chronic diseases begins not in adulthood, but during early development (1-4). This paradigm is supported by extensive human epidemiological data drawn from diverse populations, and myriad animal model data demonstrating that during critical periods of mammalian development transient nutritional stimuli can perturb developmental pathways and thereby cause persistent changes in organ structure, gene expression and metabolism. 
The specific biological mechanisms by which early nutrition causes permanent metabolic changes remain largely uncharacterized. Toward the goal of facilitating mechanistic studies in this field, the term "metabolic imprinting" was proposed to encompass adaptive responses to early nutrition characterized by (i) a persistent effect lasting into adulthood, (ii) susceptibility limited to a critical ontogenic period, (iii) a specific and measurable outcome, and (iv) a dose-response or threshold relation between exposure and outcome (3). This article will consider whether fatty acid nutrition during prenatal and early postnatal development may cause metabolic imprinting of epigenetic processes related to fatty acid metabolism.

Several recent reviews have examined the evidence that maternal fatty acid nutrition can affect metabolic development of mammalian offspring (4, 5). Most of these studies were conducted by feeding female rodents either normal or high-fat diets during pregnancy and/or lactation and examining effects in the offspring. Offspring of dams fed a high-lard diet during pregnancy and lactation develop symptoms reminiscent of metabolic syndrome, including compromised glucose homeostasis, dyslipidemia, increased blood pressure and adiposity (5). Importantly, dams fed a lard-rich diet do not increase caloric intake during pregnancy, indicating that the effects on the offspring are mediated via changes in fatty acid nutrition per se, rather than maternal overnutrition (5). The deleterious effects of the high-lard diet appear to be mediated specifically by saturated fat; offspring of rat dams fed a high-fat diet rich in polyunsaturated fatty acids (PUFAs) rather than saturated fatty acids do not become glucose intolerant (6). Importantly, the critical window for these developmental effects of maternal high-fat intake does not appear to be limited to fetal development. When rat dams are fed a high-lard diet during the suckling period only, their adult offspring develop hypertension and impaired glucose homeostasis (7). Taken together, these data indicate that the quantity and type of fatty acids consumed by the mother before and during pregnancy and lactation can induce permanent changes in the gene expression and metabolism of her offspring.

\section{Early nutrition and epigenetics}

Epigenetics describes the study of mitotically (and potentially meiotically) heritable alterations in gene expression that are not mediated by changes in DNA sequence (8). Literally meaning "above genetics", epigenetic mechanisms are gene regulatory mechanisms that are layered on top of the DNA sequence information. Diverse epigenetic mechanisms including DNA methylation, various modifications of the histone proteins that package DNA in a complex called chromatin, and autoregulatory DNA-binding proteins function synergistically to determine and maintain cell-specific gene expression patterns.

DNA methylation is the best characterized epigenetic mechanism, and is most clearly linked to nutrition (9). In mammals, DNA methylation occurs at cytosines within $\mathrm{CpG}$ dinucleotides. Tissue-specific DNA methylation patterns are established and undergo maturation during prenatal and early postnatal development and, once established, are generally maintained with high fidelity into adulthood. The methyl groups required for $\mathrm{CpG}$ methylation are derived from the diet, and several vitamins (folate, vitamins $\mathrm{B}_{6}$ and $\mathrm{B}_{12}$ ) are critical for mammalian one-carbon metabolism (10). Hence, early nutritional influences on DNA methylation (and epigenetic regulation in general) could comprise a mechanism of metabolic imprinting. Indeed, provision of extra methyl donor nutrients to female mice before and during pregnancy induces hypermethylation at the viable yellow agouti $\left(A^{v y}\right)$ locus in their offspring, with permanent phenotypic consequences (11). Establishment of gene-specific epigenetic regulation can be affected by other environmental stimuli. For example, during the first few days after birth, maternal caregiving behavior elicits changes in methylation at specific $\mathrm{CpG}$ sites of the glucocorticoid receptor $(G R)$ gene in the rat hippocampus. Consequently, relative to offspring of high-caregiving dams, those suckled by low-caregiving dams are permanently marked by $G R$ hypermethylation in the hippocampus and associated behavioral phenotypes (12). Although differing in environmental stimulus and critical window, the $A^{v y}$ and $G R$ models together illustrate an important concept: environmental influences on epigenetics occur when epigenetic regulation is undergoing developmental establishment or maturation.

\section{Fatty acids and epigenetics}

There is limited evidence that fatty acid nutrition affects epigenetic gene regulation. Although not a 
fatty acid, choline stands at the intersection of lipid and one-carbon metabolism. It is a component of phosphatidylcholine, the predominant phospholipid in mammalian cell membranes, and affects fatty acid metabolism directly by influencing the export of lipids from the liver (13). It has been proposed that choline is the predominant source of onecarbon units in the human diet (13); following conversion of choline to betaine, three methyl groups from each choline molecule can be utilized for remethylation of homocysteine to methionine. In rats, choline deficiency during fetal development causes permanent impairment of learning and memory (14). Whereas these effects could be the result of structural deficits in neuronal membranes in the central nervous system, alterations in DNA methylation have recently been implicated. Offspring of mice fed a choline-deficient diet during late gestation exhibit global and gene-specific DNA hypomethylation in specific brain regions (15). It is unknown whether the effect of choline deficiency on DNA methylation is principally mediated by effects on fatty acid or one carbon metabolism.

Studies of apolipoprotein E-deficient $\left(\right.$ Apoe $\left.^{-I-}\right)$ mice provide more direct support for the hypothesis that fatty acid nutrition, at the cellular level, can affect DNA methylation. Apolipoprotein E (ApoE) plays a critical role in the catabolism of chylomicron and very low-density lipoprotein (VLDL) remnants. Apoe ${ }^{-1-}$ mice have an increased ratio of low-density lipoprotein (LDL) plus VLDL to highdensity lipoprotein (HDL), and have been studied extensively as a mouse model of atherosclerosis. It was shown recently that the dyslipidemia in Apoe $^{-I-}$ mice correlates with DNA hypomethylation (16). In 4-week-old Apoe ${ }^{-l-}$ mice, a methylation-sensitive amplified polymorphism fingerprinting assay identified numerous loci showing hypomethylation in aorta, and these gene-specific measurements were corroborated by measurements of genome-wide methylation. To test directly the hypothesis that dyslipidemia induces hypomethylation, human monocytic THP-1 cells were cultured in media containing high or low concentrations of LDL plus VLDL to mimic the environment of aortic epithelial cells in $A p o e^{-1-}$ versus control mice (16). However, culture media high in LDL plus VLDL actually induced genome-wide hypermethylation in THP-1 cells, contrary to the effects seen in aorta of Apoe $e^{-l-}$ mice.
Although not found at appreciable levels in the diet, the short-chain fatty acid butyrate is notable for its effects on epigenetic regulation. Butyrate affects epigenetic regulation by inhibiting histone deacetylases (17). Acetylation of specific lysine residues on the tails of histone proteins contributes to the regulation of chromatin conformation and gene expression. In general, genomic regions containing hyperacetylated histones are transcriptionally active. Although not found at appreciable levels in the diet, butyrate is a by-product of fiber digestion by gut microorganisms, and is the preferred energy source of colonocytes. It has been proposed that dietary fiber may protect against colon cancer via the effect of butyrate on epigenetic gene regulation (17). The specific mechanism by which butyrate inhibits histone deacetylases remains unknown. Nonetheless, its powerful influence on epigenetic regulation raises the possibility that fatty acids found more commonly in the diet could directly affect epigenetic processes. Although not a fatty acid, cholesterol is intimately related to fatty acid metabolism and merits mention here; limited data indicate that cholesterol acts as an inhibitor of DNA methyltransferases, the enzymes responsible for establishing and maintaining CpG methylation (18).

Overall, the data indicating that dietary fatty acids have a direct impact on epigenetic processes are not compelling. Most studies of early nutritional influences on epigenetic regulation have focused on dietary exposures that affect one-carbon metabolism, with the goal of inducing secondary effects on DNA methylation and overall epigenetic gene regulation. A less obvious but potentially more important mechanism by which early nutrition may affect the establishment of epigenetic regulation is by influencing transcriptional activity of specific genes during critical ontogenic periods. It has long been known that promoter region hypermethylation can affect the binding of methyl-sensitive DNA binding proteins and thereby contribute to transcriptional silencing. It has more recently become clear that transcriptional activity can serve as the basis for epigenetic memory (19). One model for the establishment of tissue-specific DNA methylation patterns during development proposes that, in different tissue lineages, genes being actively transcribed are protected from de novo methylation (8). By this model, any nutrient that can induce transcriptional activation of specific genes could have an important influence on the developmental 
establishment of epigenetic regulation. The following example considers the potential for fatty acid nutrition during development to cause persistent changes in fatty acid metabolism by inducing epigenetic changes at the gene encoding the peroxisome proliferator-activated receptor (PPAR)- $\alpha$.

\section{PPAR- $\alpha$ in fatty acid metabolism}

PPARs are among the most abundant classes of nuclear receptor transcription factors and play a critical role in the regulation of fatty acid metabolism. PPAR- $\alpha$ was the first PPAR to be identified and is expressed mainly in tissues with high rates of fatty acid oxidation, including liver, heart, and skeletal muscle (20). PPAR- $\alpha$ is localized in the cytoplasm in association with heat shock protein 90 (HSP90). Binding of medium- and long-chain PUFAs to PPAR- $\alpha$ induces a conformational change in PPAR $-\alpha$ and its dissociation from HSP90. PPAR- $\alpha$ then translocates to the nucleus, where it heterodimerizes with other nuclear receptors such as the retinoid $\mathrm{X}$ receptor ( $\mathrm{RXR}$ ) and alters the expression of target genes by transactivating the peroxisome proliferator response element (PPRE) (21). Several key enzymes in fatty acid metabolism contain PPRE and therefore respond to changes in PUFA levels in a PPAR- $\alpha$-dependent manner. For example, the genes encoding acyl-coenzyme A oxidase (Aox) and cytochrome p450 4A2 (Cyp4A2) are regulated in this manner. Expression of Aox and Cyp $4 A 2$ increases in response to dietary PUFA in wild-type but not in PPAR $-\alpha^{-1-}$ mice (22).

\section{PPAR- $\alpha$ genetics and epigenetics}

Several common polymorphisms have been identified in the coding region of $P P A R-\alpha$ gene. The $\mathrm{L} 162 \mathrm{~V}$ polymorphism is of particular interest; humans with this missense variant have dyslipidemia comprising elevated plasma cholesterol, HDL-cholesterol and Apo $A_{\text {I }}$ levels (20). The PPAR- $\alpha$ polymorphism D22S928, although $1 \mathrm{Mb}$ upstream of the PPAR- $\alpha$ promoter, is associated with familial hyperlipidemia (23). Just as genetic mutations can lead to disease, so too can epimutations; this assertion is now well established with respect to cancer (24). Analogously, epigenetic dysregulation could cause permanent changes in the expression of PPAR- $\alpha$. Hence, in addition to genetic polymorphisms, interindividual variations in epigenetic regulation at $P P A R-\alpha$ could affect susceptibility to dyslipidemia.

\section{Tissue-specific PPAR- $\alpha$ ontogeny}

Examining the ontogeny of PPAR- $\alpha$ expression provides insight into the development of its tissuespecific epigenetic regulation. In the rat, PPAR- $\alpha$ mRNA is first detected around embryonic day 13.5 (E13.5) in the liver, renal cortex, intestinal mucosa, pancreas, and heart (25). In the developing rat liver, PPAR- $\alpha$ mRNA expression is robust and fairly stable from E19 to postnatal day 7 (P7), then declines significantly in adulthood (26). In rat heart, PPAR- $\alpha$ shows a markedly different ontogeny, with expression increasing significantly from $\mathrm{P} 0$ to $\mathrm{P} 21$ (27). PPAR- $\alpha$ expression remains undetectable in mouse muscle at E19, then peaks dramatically at birth before diminishing to very low levels by P21 (28). It makes sense that PPAR- $\alpha$ expression changes around the time of birth, stimulated by the nutritional transition from the high-carbohydrate diet of the fetus to the high-fat diet of the suckling mammal. The tissue specificity of its perinatal ontogeny suggests that epigenetic regulation of PPAR- $\alpha$ is established differently in various tissues during prenatal development and continues to mature in the early postnatal period. Experiments examining the transcriptional activation of PPAR $-\alpha$ in response to dietary fatty acids further support this conjecture. In 10-day-old rats, hepatic PPAR- $\alpha$ expression is up-regulated in response to elevations in plasma free fatty acids (FFAs); this responsiveness is not detected in adult liver (29).

\section{Maternal diet and PPAR- $\alpha$ expression}

As discussed above, active transcription of a gene during development can inhibit the establishment of DNA methylation at the locus. The transcriptional activation of PPAR- $\alpha$ around the time of birth thus raises the question of whether maternal diet affects PPAR- $\alpha$ expression in the fetus or suckling infant. In humans, maternal PUFA intake affects PUFA concentrations in the newborn. Newborn infants of mothers supplemented with fish oil in the third trimester of pregnancy exhibit significantly higher plasma 22:6 n-3 (docosahexanoic acid) concentrations than those not supplemented or supplemented with olive oil (30). Recent studies in sheep show that maternal nutrition during gestation affects fetal adipose tissue PPAR- $\alpha$ mRNA expression (31). In that model, pregnant ewes were calorically restricted during early pregnancy then refed during late pregnancy. Following the period of refeeding, fetuses of restricted ewes had elevated adipose tissue mass 
and adipose PPAR- $\alpha$ mRNA expression relative to fetuses of unrestricted mothers. Studies in the rat show that PPAR- $\alpha$ methylation in the offspring is affected by maternal diet. At weaning, hepatic PPAR- $\alpha$ methylation in offspring of protein-restricted mothers was lower than that of unrestricted offspring. PPAR- $\alpha$ hypomethylation correlated with a significant increase in PPAR- $\alpha$ expression (32).

Together, these data suggest that the epigenetic regulation of PPAR- $\alpha$ is undergoing developmental changes in the late fetal and early postnatal period in mammals, and that these mechanisms can be influenced by maternal diet. There are currently no data, however, showing that maternal nutrition during pregnancy and/or lactation induces persistent changes in PPAR- $\alpha$ expression in the offspring.

\section{Conclusion and suggestions for future research}

Animal data show that fatty acid nutrition during prenatal and early postnatal development can have a persistent influence on metabolism. Nutritional influences on developmental epigenetics are one potential mechanism to explain these phenomena. Fatty acid nutrition during development could affect the establishment of epigenetic gene regulation by inducing (or repressing) transcription of specific genes during critical ontogenic periods during which tissue-specific epigenetic marks are initiated. Hence, it is critically important to characterize the developmental epigenetics at genes playing central roles in mammalian fatty acid metabolism. The ontogenic periods during which tissue-specific epigenetic marks are established at these genes constitute likely critical periods during which epigenetically mediated metabolic imprinting of fatty acid metabolism could occur.

\section{Acknowledgements}

RAW is supported by NIH grant 5K01DK070007, research grant no. 5-FY05-47 from the March of Dimes Birth Defects Foundation, and USDA CRIS no. 6250-51000-049.

\section{References}

1. Dorner G. Environment-dependent brain differentiation and fundamental processes of life. Acta Biol Med Ger 1974; 33: 129-48.

2. Barker DJ. Programming the baby. In: Barker DJ, ed. Mothers, babies, and disease in later life. London: BMJ Publishing Group; 1994. p. 14-36.
3. Waterland RA, Garza C. Potential mechanisms of metabolic imprinting that lead to chronic disease. Am J Clin Nutr 1999; 69: 179-97.

4. McMillen IC, Robinson JS. Developmental origins of the metabolic syndrome: prediction, plasticity, and programming. Physiol Rev 2005; 85: 571-633.

5. Armitage JA, Taylor PD, Poston L. Experimental models of developmental programming: consequences of exposure to an energy rich diet during development. $\mathbf{J}$ Physiol 2005; 565(Pt 1): 3-8.

6. Siemelink M, Verhoef A, Dormans JA, Span PN, Piersma AH. Dietary fatty acid composition during pregnancy and lactation in the rat programs growth and glucose metabolism in the offspring. Diabetologia 2002; 45: $1397-403$.

7. Khan IY, Dekou V, Douglas G, Jensen R, Hanson MA, Poston L, et al. A high-fat diet during rat pregnancy or suckling induces cardiovascular dysfunction in adult offspring. Am J Physiol Regul Integr Comp Physiol 2005; 28: R127-33.

8. Jaenisch R, Bird A. Epigenetic regulation of gene expression: how the genome integrates intrinsic and environmental signals. Nat Genet 2003; 33(Suppl): 245-54.

9. Waterland RA, Jirtle RL. Early nutrition, epigenetic changes at transposons and imprinted genes, and enhanced susceptibility to adult chronic diseases. Nutrition 2004; 20: 63-8.

10. Van den Veyver I. Genetic effects of methylation diets. Annu Rev Nutr 2002; 22: 255-82.

11. Waterland RA, Jirtle RL. Transposable elements: targets for early nutritional effects on epigenetic gene regulation. Mol Cell Biol 2003; 23: 5293-300.

12. Weaver IC, Cervoni N, Champagne FA, D'Alessio AC, Sharma S, Seckl JR, et al. Epigenetic programming by maternal behavior. Nat Neurosci 2004; 7: 847-54.

13. Zeisel SH. Choline, homocysteine, and pregnancy. Am J Clin Nutr 2005; 82: 719-20.

14. Meck WH, Williams CL. Metabolic imprinting of choline by its availability during gestation: implications for memory and attentional processing across the lifespan. Neurosci Biobehav Rev 2003; 27: 385-99.

15. Niculescu MD, Craciunescu CN, Zeisel SH. Dietary choline deficiency alters global and gene-specific DNA methylation in the developing hippocampus of mouse fetal brains. FASEB J 2006; 20: 43-9.

16. Lund G, Andersson L, Lauria M, Lindholm M, Fraga MF, Villar-Garea A, et al. DNA methylation polymorphisms precede any histological sign of atherosclerosis in mice lacking apolipoprotein E. J Biol Chem 2004; 279: 29147-54.

17. Davie JR. Inhibition of histone deacetylase activity by butyrate. J Nutr 2003; 133: 2485-93S.

18. Zaina S, Dossing KB, Lindholm MW, Lund G. Chromatin modification by lipids and lipoprotein components: an initiating event in atherogenesis? Curr Opin Lipidol 2005; 16: 549-53.

19. Ng RK, Gurdon JB. Epigenetic memory of active gene transcription is inherited through somatic cell nuclear transfer. Proc Natl Acad Sci U S A 2005; 102: 1957-62. 
20. Vohl MC, Lepage P, Gaudet D, Brewer CG, Betard C, Perron $\mathrm{P}$, et al. Molecular scanning of the human PPARa gene: association of the L162v mutation with hyperapobetalipoproteinemia. J Lipid Res 2000; 41: 945-52.

21. Sumanasekera WK, Tien ES, Turpey R, Vanden Heuvel JP, Perdew GH. Evidence that peroxisome proliferatoractivated receptor alpha is complexed with the $90-\mathrm{kDa}$ heat shock protein and the hepatitis virus $\mathrm{B}$ X-associated protein 2. J Biol Chem 2003; 278: 4467-73.

22. Ren B, Thelen AP, Peters JM, Gonzalez FJ, Jump DB. Polyunsaturated fatty acid suppression of hepatic fatty acid synthase and S14 gene expression does not require peroxisome proliferator-activated receptor alpha. J Biol Chem 1997; 272: 26827-32.

23. Eurlings PM, van der Kallen CJ, Geurts JM, Flavell DM, de Bruin TW. Identification of the PPARA locus on chromosome 22q13.3 as a modifier gene in familial combined hyperlipidemia. Mol Genet Metab 2002; 77: 274-81.

24. Jones PA, Baylin SB. The fundamental role of epigenetic events in cancer. Nat Rev Genet 2002; 3: 415-28.

25. Braissant O, Wahli W. Differential expression of peroxisome proliferator-activated receptor-alpha, -beta, and gamma during rat embryonic development. Endocrinology 1998; 139: 2748-54.

26. Gruppuso PA, Boylan JM, Vaslet CA. Identification of candidate growth-regulating genes that are overexpressed in late gestation fetal liver in the rat. Biochim Biophys Acta 2000; 1494: 242-7.

27. Steinmetz M, Quentin T, Poppe A, Paul T, Jux C. Changes in expression levels of genes involved in fatty acid metabolism: upregulation of all three members of the PPAR family (alpha, gamma, delta) and the newly described adiponectin receptor 2 , but not adiponectin receptor 1 during neonatal cardiac development of the rat. Basic Res Cardiol 2005; 100: 263-9.
28. Brun S, Carmona MC, Mampel T, Vinas O, Giralt M, Iglesias R, et al. Activators of peroxisome proliferatoractivated receptor-alpha induce the expression of the uncoupling protein-3 gene in skeletal muscle: a potential mechanism for the lipid intake-dependent activation of uncoupling protein-3 gene expression at birth. Diabetes 1999; 48: 1217-22.

29. Panadero M, Herrera E, Bocos C. Different sensitivity of PPARalpha gene expression to nutritional changes in liver of suckling and adult rats. Life Sci 2005; 76: $1061-$ 72.

30. Hornstra G. Essential fatty acids in mothers and their neonates. Am J Clin Nutr 2000; 71: 1262-9S.

31. Bispham J, Gardner DS, Gnanalingham MG, Stephenson T, Symonds ME, Budge H. Maternal nutritional programming of fetal adipose tissue development: differential effects on messenger ribonucleic acid abundance for uncoupling proteins and peroxisome proliferator-activated and prolactin receptors. Endocrinology 2005; 146: 3943-9.

32. Lillycrop KA, Phillips ES, Jackson AA, Hanson MA, Burdge GC. Dietary protein restriction of pregnant rats induces and folic acid supplementation prevents epigenetic modification of hepatic gene expression in the offspring. J Nutr 2005; 135: 1382-6.

Robert A. Waterland, PhD

Baylor College of Medicine, Departments of Pediatrics and Molecular and Human Genetics USDA Children's Nutrition Research Center I I00 Bates Ave. Ste. 5080

Houston TX 77030 USA

Tel: + I 7137980304

Fax: + 7137987101

E-mail: waterland@bcm.edu 